\title{
Angiographic Patterns of In-Stent Restenosis and Implications on Subsequent Revascularization
}

\author{
Annapoorna Kini, MD, Jonathan D. Marmur, MD, George Dangas, MD, PhD, \\ Sanjay Choudhary, MD, and Samin K. Sharma,* MD
}

\begin{abstract}
Stent implantation has become the mainstay of percutaneous revascularization for most coronary lesions; in-stent restenosis (ISR) can occur in $6 \%-40 \%$ of stent procedures and the subsequent response to repeat intervention can possibly be predicted by the angiographic patterns of ISR. This study evaluated the incidence and predictors of angiographic patterns of ISR and its impact on subsequent target lesion revascularization (TLR) in 100 consecutive patients having Palmaz-Schatz ISR undergoing intervention. Diffuse ISR ( $\geq 10 \mathrm{~mm}$ ) was observed in $78 \%$ and focal ISR $(>10 \mathrm{~mm})$ in $22 \%$. Diffuse vs. focal ISR occurred earlier after stent implantation and was more common in diabetics. Angiographic predictors of diffuse ISR were stent implantation for a restenotic lesion, long lesions, smaller vessel, stenting without debulking, and high-pressure balloon inflation ( $>16 \mathrm{~atm})$. TLR after repeat intervention was $46 \%$ for diffuse and $14 \%$ for focal ISR $(P<0.02)$. Rotational atherectomy resulted in lower TLR $(31 \%)$ vs. PTCA or restent $(64 \%)$ in diffuse ISR $(P<0.004)$. Therefore, diffuse ISR is more common than focal ISR, usually occurs in the setting of aggressive intimal hyperplasia, and can be predicted by clinical and angiographic variables. Also, diffuse intimal hyperplasia within a stent responds poorly to PTCA and may benefit from a more aggressive debulking strategy such as rotational atherectomy. Cathet. Cardiovasc. Intervent. 49:23-29, 2000. ๑ 2000 Wiley-Liss, Inc.
\end{abstract}

Key words: diffuse in-stent restenosis; target lesion revascularization; rotational atherectomy

\section{INTRODUCTION}

Coronary stenting has been shown to improve both short- and long-term results of percutaneous interventions of various coronary lesions and makes up to $90 \%$ of the procedures at some centers [1,2]. Restenosis after stent implantation commonly labeled as in-stent restenosis (ISR) poses a special challenge to interventional cardiologists. ISR has been reported to occur in $6 \%-40 \%$ of cases based on the lesion length, vessel size, postprocedure minimal lumen diameter (MLD), and clinical factors such as diabetes mellitus and unstable angina [3-6]. Numerous intravascular ultrasound (IVUS) and histopathologic data have revealed intimal hyperplasia as the sole cause of ISR after properly deployed tubular stents (such as PalmazSchatz) and stent struts are not compressed [7,8]. Angiographically (Fig. 1), ISR has traditionally been classified as either focal $(<10 \mathrm{~mm}$ in length occurring within the stent, at edges or articulation site), or diffuse ( $\geq 10 \mathrm{~mm}$ in length, usually covering entire stent length or even extending outside into the nonstented segment). The factors leading to diffuse vs. focal ISR after stent implantation have not been well characterized [9-11]. The angiographic pattern of ISR may have important implications on subsequent outcome after repeat interven- tion because diffuse ISR has been predicted to have a higher subsequent restenosis after balloon angioplasty (PTCA) $[9,12,13]$. The short- and long-term results of other interventional modalities such as rotational atherectomy (PRCA), directional atherectomy, excimer laser, or restenting for ISR treatment is not clear and the angiographic pattern of ISR may be an important factor in selecting the devices for the intervention [14-17]. The present study was therefore designed to evaluate the incidence and predictors of focal vs. diffuse pattern of ISR after Palmaz-Schatz stent implantation for single vessel disease, and to assess the long-term outcome after reintervention using a variety of interventional devices.

Cardiac Catheterization Laboratory, the Zena \& Michael A. Wiener Cardiovascular Institute, Mount Sinai Hospital, New York

Presented in part at the 46th annual scientific session of the American College of Cardiology in Anaheim, CA, March 1997, and the 19th Congress of the European Society of Cardiology in Stockholm, Sweden, August 1997.

*Correspondence to: Dr. Samin K. Sharma, Mount Sinai Hospital, Box 1030, One Gustave Levy Place, New York, NY 10029.

E-mail: sharma@smtplink.mssm.edu

Received 6 April 1999; Revision accepted 30 June 1999 
Focal $(<10 \mathrm{~mm})$ ISR

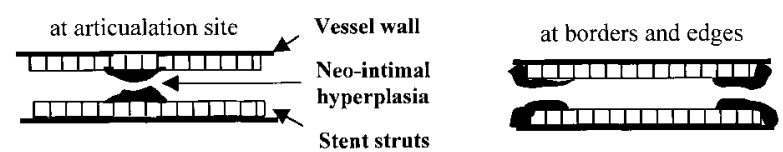

Diffuse ( $>10 \mathrm{~mm})$ ISR

Intra-stent in most part of the stent

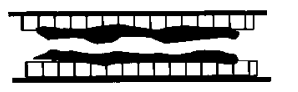

Extending beyond the stent

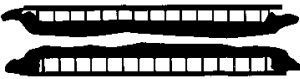

Fig. 1. Schematic outline of different angiographic patterns of in-stent restenosis (ISR).

\section{MATERIALS AND METHODS Patient Population}

One hundred and nine consecutive patients with native single-vessel in-stent restenosis ( $>50 \%$ diameter obstruction occurring 8 weeks poststenting) after high-pressure $\geq 3.0 \mathrm{~mm}$ Palmaz-Schatz stent implantation undergoing reintervention at the Mount Sinai Hospital, New York, from January 1995 to August 1996, were studied. Of these, eight patients presented with total occlusion and were excluded from the analysis. A total of 101 consecutive patients comprised the study population. All of these patients were either symptomatic $(n=92)$ or had significant evidence of ischemia on noninvasive testing $(\mathrm{n}=9)$. Clinical, angiographic, and procedural data and cine angiograms of the initial stent implantation as well as the current intervention were available in all patients.

\section{Intervention}

All patients were informed regarding the coronary interventional procedure and a written consent was obtained. All patients received aspirin $325 \mathrm{mg}$ orally and $100-\mathrm{U} / \mathrm{kg}$ intravenous bolus of heparin. The activated clotting time was maintained between 250-300 sec throughout the procedure using periodic intravenous boluses of heparin. The choice of the intervention was left at the operator's discretion and included PTCA, PRCA, or restenting. A 7-10 Fr guiding catheter was used. An oversized non-compliant balloon (1.1-1.2:1 balloon-toartery ratio) at $12-16 \mathrm{~atm}$ for $60-120 \mathrm{sec}$ was initially used for PTCA. In cases of suboptimal results, a larger balloon was used to achieve the desired results. In cases of edge dissection, intimal dissection within the stent, or suboptimal angiographic results, an additional stent was implanted. For RE-STENT, stent was not used as a primary modality for ISR treatment and was only used in cases of focal or articulation defect not responding well to high-pressure PTCA or as a bail out after PTCA. For
PRCA, rotational atherectomy was initiated in the middle of the study period (November 1995) in the treatment of ISR. Step burr approach was used in all cases and a final burr-to-artery (BA) ratio of $>0.70$ was targeted. In the initial cases, BA ratio was lower $(<0.60)$, but with experience, and absence of the usual complications associated with PRCA (such as slow flow and hypotension), a higher BA ratio of $\sim 0.70$ was attained in the later cases (mean $0.72 \pm 0.12$ ). After PRCA, all lesions were postdilated with a balloon at 3-6 atm for $60-90 \mathrm{sec}$. After PRCA, the angiographic lumen was smoother without any dissection and did not require stent implantation. IVUS was performed in initial PRCA cases at baseline, postablation, and postprocedure to understand the mechanism of luminal enlargement postablation.

\section{Postprocedure Care}

Sheaths were removed 4-6 hr after intervention. ReoPro was used in less than $10 \%$ of cases. CK-MB was measured preprocedure, 8 and $16 \mathrm{hr}$ postprocedure, and patients were discharged the next day, on aspirin only after PTCA and PRCA and on aspirin and ticlopidine 250 mg twice daily for 4 weeks if another stent was implanted.

\section{Follow-Up}

All patients were followed up clinically by telephone contact or physician visit at a mean duration of $13 \pm 4$ months, with a minimum of 9 months. Clinical cardiac events and need for repeat target vessel revascularization (TLR) was determined in all patients.

\section{Angiographic Analysis}

A single operator (SC) using previously validated system of quantitative coronary angiography by the Computerized Medical System (CMS, Medis, The Netherlands) analyzed all cine angiogram using the contrast filled catheter as the calibration standard [18]. Final results were the average of the two orthogonal views with the least amount of foreshortening and overlap. Reference vessel diameter (RVD), minimal lumen diameter (MLD), and percent diameter stenosis (DS) were measured. Lesion length was measured by the computerized edge detection method. Slow flow was assessed visually and a single operator (SS) classified lesions according to the standard angiographic definitions.

\section{Definitions}

Focal ISR denotes restenotic lesion $<10 \mathrm{~mm}$ in length anywhere within the stent; diffuse ISR, restenotic lesion $\geq 10 \mathrm{~mm}$ in length within the stent and may continue through the stent edges into the nonstented vessel segment; angiographic success, $<30 \%$ diameter obstruction postprocedure; clinical success, angiographic success and 
no major complications (Q-wave myocardial infarction, emergent bypass surgery, or death); acute closure, postprocedure out of the catheterization laboratory occlusion in the treated vessel; slow flow, delayed distal clearance of the dye in the absence of proximal dissection or spasm; dissection, coronary dissection were graded from $\mathrm{A}$ to $\mathrm{F}$ according to the NHLBI classification and dissection grade $\mathrm{A}$ to $\mathrm{C}$ were considered minor and grade $\mathrm{D}$ to $\mathrm{F}$ were considered major; calcification, readily apparent fluoroscopic densities in the lesion or vessel; vascular complications, large hematoma $\left(>3 \times 3^{\prime \prime}\right.$ in size), bleeding requiring transfusion, pseudoaneurysm, or need for vascular surgery.

\section{Statistics}

Results are presented as mean \pm SD or $\mathrm{n}(\%)$. Comparison between two groups was done with the Fisher exact test or chi-square analysis for categorical variables and two-tail Student's $t$-test for continuos variables. Statistical significance was defined at the level of $P<0.05$. The data were entered in a FileMaker Pro 2.1 (Claris) database and transferred to the statistical program StatView 4.1 for analysis. The contribution of clinical, angiographic, and procedural variables to the type of ISR was calculated by univariate analysis and by Cox multiple logistic regression model, where selection of the variables was achieved in a stepwise fashion and data are presented as odds ratio (OR) and 95\% confidence interval (CI) with a significant $P$ value of $<0.05$.

\section{RESULTS}

Of the 101 cases, diffuse ISR occurred in 79 patients $(78 \%)$ and focal ISR in $22(22 \%)$. Focal ISR occurred equally at the articulation site (50\%) and at stent edges (50\%). In 18 patients (18\%) with diffuse ISR, restenotic lesion extended $>5 \mathrm{~mm}$ from stent edges into the nonstented vessel.

\section{Clinical and Procedural Characteristics at Time of Initial Stent Deployment}

A total of 44 clinical, angiographic, and procedural variables at the time of stent deployment were compared between the two groups (Table I). Univariate predictors of diffuse ISR were diabetes mellitus, restenosis lesion, long lesion length, ACC/AHA type C lesion, multiple stents, debulking prior to stenting, inflation pressure $>16$ atm, and smaller reference vessel diameter. On multivariate logistic regression analysis, the following factors independently correlated with ISR: diabetes mellitus (OR $2.2,95 \%$ CI 1.6-4.2; $P=0.02$ ), restenotic lesion (OR $2.1,95 \%$ CI $1.3-4.1 ; P=0.03)$, long lesion length (OR $4.5,95 \%$ CI $2.3-7.2 ; P<0.001)$, inflation pressure $>16$ atm (OR 3.2, 95\% CI 1.2-4.8; $P=0.02$ ), and stenting
TABLE I. Various Clinical, Angiographic, and Procedural Characteristics at the Time of Initial Stent Deployment Between Two Groups*

\begin{tabular}{lccc}
\hline Variables & $\begin{array}{c}\text { Focal } \\
(\mathrm{n}=22)\end{array}$ & $\begin{array}{c}\text { Diffuse } \\
(\mathrm{n}=79)\end{array}$ & $P$ \\
\hline Age (years) & $62 \pm 18$ & $65 \pm 12$ & NS \\
CCS class III-IV (\%) & $15(68)$ & $46(59)$ & NS \\
Diabetes mellitus (\%) & $5(17)$ & $34(43)$ & 0.04 \\
Multivessel disease (\%) & $10(45)$ & $44(56)$ & NS \\
LVEF (\%) & $52 \pm 18$ & $48 \pm 13$ & NS \\
Restenotic lesion (\%) & $3(14)$ & $26(33)$ & 0.05 \\
ReoPro use (\%) & $5(23)$ & $23(29)$ & NS \\
Lesion length (mm) & $12 \pm 4$ & $17 \pm 8$ & 0.02 \\
ACC/AHA type C lesion (\%) & $3(14)$ & $29(37)$ & 0.03 \\
Moderate/heavy calcification (\%) & $4(18)$ & $21(27)$ & NS \\
Total occlusion (\%) & $2(9)$ & $6(8)$ & NS \\
Multiple stents (\%) & $4(18)$ & $41(52)$ & 0.01 \\
Complex lesion (\%) & $6(27)$ & $25(32)$ & NS \\
Inflation pressure $>16$ atm (\%) & $6(27)$ & $52(66)$ & $<0.001$ \\
Debulking prior to stenting (\%) & $15(68)$ & $25(32)$ & $<0.001$ \\
Maximal balloon size (mm) & $3.4 \pm 0.8$ & $3.3 \pm 0.7$ & 0.1 \\
Reference vessel diameter (mm) & $3.26 \pm 0.31$ & $3.11 \pm 0.52$ & 0.03 \\
Preprocedure MLD (mm) & $0.86 \pm 0.38$ & $0.81 \pm 0.41$ & NS \\
Postprocedure MLD (mm) & $3.10 \pm 0.42$ & $3.04 \pm 0.32$ & NS \\
\hline
\end{tabular}

*CCS, Canadian Cardiovascular Society; LVEF, left ventricular enjection fraction; ACC/AHA, American College of Cardiology/American Heart Association; MLD, minimal lumen diameter.

aDebulking device, 94\% PRCA and 6\% directional coronary atherectomy.

TABLE II. Baseline Clinical Characteristics Between Two Groups*

\begin{tabular}{lccc}
\hline Variables & $\begin{array}{c}\text { Focal ISR } \\
(\mathrm{n}=22)\end{array}$ & $\begin{array}{c}\text { Diffuse ISR } \\
(\mathrm{n}=79)\end{array}$ & $P$ \\
\hline Age (years) & $64 \pm 17$ & $66 \pm 15$ & NS \\
Male (\%) & $17(77)$ & $63(80)$ & NS \\
CCS class III-IV (\%) & $11(50)$ & $38(48)$ & NS \\
Post MI (\%) & $2(9)$ & $9(11)$ & NS \\
Diabetes mellitus (\%) & $6(27)$ & $35(44)$ & 0.07 \\
Hypercholesterolemia (\%) & $10(45)$ & $42(53)$ & NS \\
Hypertension (\%) & $7(32)$ & $31(39)$ & NS \\
Smoking (\%) & $5(23)$ & $9(11)$ & NS \\
Multivessel disease (\%) & $5(23)$ & $21(27)$ & NS \\
LVEF $<30(\%)$ & $5(23)$ & $16(20)$ & NS \\
Time interval for ISR (in days) & $161 \pm 47$ & $129 \pm 38$ & 0.008 \\
\hline
\end{tabular}

*CCS, Canadian Cardiovascular Society; LVEF, left ventricular ejection fraction; MI, myocardial infarction.

without debulking (OR 4.5, 95\% CI 3.1-7.8; $P<0.001)$. Other factors such as age, CCS angina class III-IV, multivessel disease, left ventricular ejection fraction, total occlusion, ReoPro use, preprocedure and postprocedure MLD did not correlate with the diffuse ISR.

\section{Clinical Characteristics}

The mean age of the study population was $66 \pm 16$ years with 79\% males (Table II). The Canadian Cardiovascular Society class III-IV angina was the presenting symptom in $50 \%$ of cases and $11 \%$ were postmyocardial infarction. The mean left ventricular ejection fraction 
Table III. Angiographic and Procedural Characteristics Between Two Groups*

\begin{tabular}{lccc}
\hline Variables & $\begin{array}{c}\text { Focal ISR } \\
(\mathrm{n}=22)\end{array}$ & $\begin{array}{c}\text { Diffuse ISR } \\
(\mathrm{n}=79)\end{array}$ & $P$ \\
\hline Vessel involved $(\%)$ & & & \\
$\quad$ LAD & $8(36)$ & $45(57)$ & 0.08 \\
RCA & $10(46)$ & $27(34)$ & $\mathrm{NS}$ \\
LCX & $4(18)$ & $7(9)$ & $\mathrm{NS}$ \\
Lesion length (mm) & $8 \pm 3$ & $17 \pm 5$ & $<0.001$ \\
Complex lesion $(\%)$ & $3(14)$ & $15(19)$ & $\mathrm{NS}$ \\
ACC/AHA type C lesions $(\%)$ & $2(9)$ & $28(35)$ & 0.01 \\
Reference vessel diameter (mm) & $3.21 \pm 0.22$ & $3.09 \pm 0.31$ & 0.05 \\
Preprocedure MLD (mm) & $0.72 \pm 0.23$ & $0.78 \pm 0.18$ & $\mathrm{NS}$ \\
Postprocedure MLD (mm) & $2.91 \pm 0.32$ & $2.85 \pm 0.71$ & $\mathrm{NS}$ \\
Angiographic success $(\%)$ & $22(100)$ & $79(100)$ & $\mathrm{NS}$ \\
Clinical success $(\%)$ & $22(100)$ & $79(100)$ & $\mathrm{NS}$ \\
Minor dissection $(\%)$ & $1(4.5)$ & $3(3.8)$ & $\mathrm{NS}$ \\
Side branch closure $(\%)$ & $0(0)$ & $2(2.5)$ & $\mathrm{NS}$ \\
Slow flow $(\%)$ & $0(0)$ & $2(2.5)$ & $\mathrm{NS}$ \\
CK-MB elevation $(\%)$ & $2(9.1)$ & $9(11.4)$ & $\mathrm{NS}$ \\
$\quad$ CK-MB $>3 \times$ control $(\%)$ & $0(0)$ & $2(2.5)$ & NS \\
\hline
\end{tabular}

*LAD, left anterior descending; LCX, left circumflex; MLD, minimal lumen diameter; RCA, right coronary artery; $\mathrm{CK}-\mathrm{MB}$, creatine kinase MB isoenzyme.

(LVEF) was 50\% (21\% had LVEF < 30\%). Mean time interval for ISR was $161 \pm 47$ days for focal and $129 \pm$ 38 days for diffuse pattern $(P=0.008)$. There was a trend toward higher incidence of diabetes mellitus in the diffuse group (44\% vs. $27 \%$ in focal; $P=0.07$ ). Other factors like smoking, hypertension, and multivessel disease were not different between the two groups.

\section{Angiographic and Procedural Characteristics}

There was a trend towards higher LAD involvement in diffuse ISR compare to focal ISR $(57 \%$ vs. $36 \% ; P=$ 0.08 ; Table III). The mean lesion length was $8 \pm 3 \mathrm{~mm}$ for focal and $17 \pm 5 \mathrm{~mm}$ for diffuse type $(P<0.001)$ with higher ACC/AHA type $\mathrm{C}$ lesions in the diffuse ISR group (35\% vs. $9 \%$ in focal; $P=0.01$ ). All procedures $(100 \%)$ were successful angiographically and clinically without any major complications. There were no major angiographic dissections. Minor angiographic dissections were noted in $4 \%$, slow flow in $2 \%$, and side-branch occlusion in $2 \%$, and were not different between the two groups. Any CK-MB elevation was $9.1 \%$ in focal and $11.4 \%$ in the diffuse pattern $(P=\mathrm{NS})$. There were no vascular complications and all patients were discharged home after the intervention at a mean duration of $1.7 \pm 1.1$ days. Quantitative coronary analysis revealed pre- and postprocedure MLD to be similar between the two groups. The final postprocedure MLD after ISR treatment was smaller $(\sim 0.2 \mathrm{~mm})$ than the postprocedure MLD achieved after initial stent deployment $(2.87 \pm 0.28 \mathrm{~mm}$ vs. $3.08 \pm 0.32 ; P=0.02)$. Of the 101 patients, $32 \mathrm{had}$

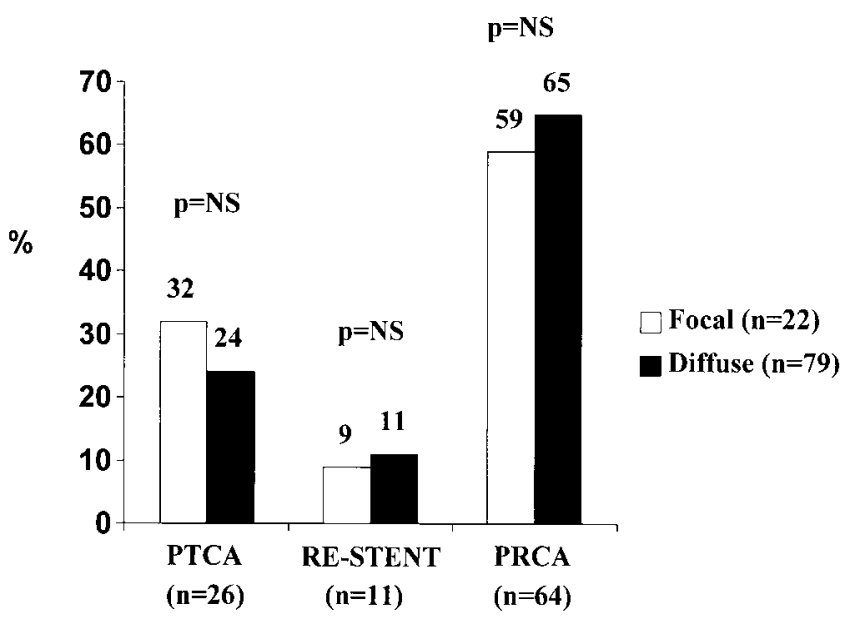

Fig. 2. Interventional device use in two different angiographic patterns of in-stent restenosis (ISR). PTCA = balloon angioplasty; PRCA = rotational atherectomy.

multiple stents in vessel size $\leq 3.0 \mathrm{~mm}$ and in-stent restenosis was of diffuse type in $91 \%$ of cases $(n=29)$.

\section{Type of Intervention}

PTCA was used in 26 patients $(26 \%)$, restent in 11 patients $(11 \%)$, and PRCA in 64 patients $(63 \%)$. The interventional devices used were not different between the two angiographic patterns (Fig. 2). Postprocedure MLD was larger after PRCA compared to PTCA (2.98 \pm $0.29 \mathrm{~mm}$ vs. $2.76 \pm 0.24 \mathrm{~mm} ; P=0.01)$.

\section{Follow-Up}

Clinical follow-up at a mean of $13 \pm 4$ months was complete in all cases $(100 \%)$ and all patients are alive except for one sudden death 5 months after intervention in the diffuse group. In the focal group, one patient presented with non-Q-wave myocardial infarction due to repeat in-stent occlusion. In the diffuse group, one patient presented with uncomplicated Q-wave myocardial infarction and one with non-Q-wave myocardial infarction due to repeat in-stent occlusion. The clinical restenosis (Fig. 3 ), defined as $>50 \%$ diameter stenosis on angiography or need for TLR, occurred in 36 patients $(46 \%)$ in the diffuse group and in 3 patients $(14 \%)$ in the focal group $(P=$ 0.02 ). The impact of different devices on subsequent TLR after treatment of diffuse ISR is shown in Figure 4: $31 \%$ after PRCA vs. 64\% after PTCA and restenting $(P<$ 0.004). The multivariate predictors of lower TLR after ISR treatment were final MLD (OR 3.1, 95\% CI 2.8-3.7; $P=0.01)$ and PRCA use (OR 4.2, 95\% CI 2.8-7.6; $P<$ 0.001 ), while diabetes mellitus was not predictive (OR 1.13 ; 95\% CI 0.87-1.30; $P=0.3$ ). During the follow-up period, two patients in the focal group and nine patients in the diffuse group required nontarget vessel intervention. 


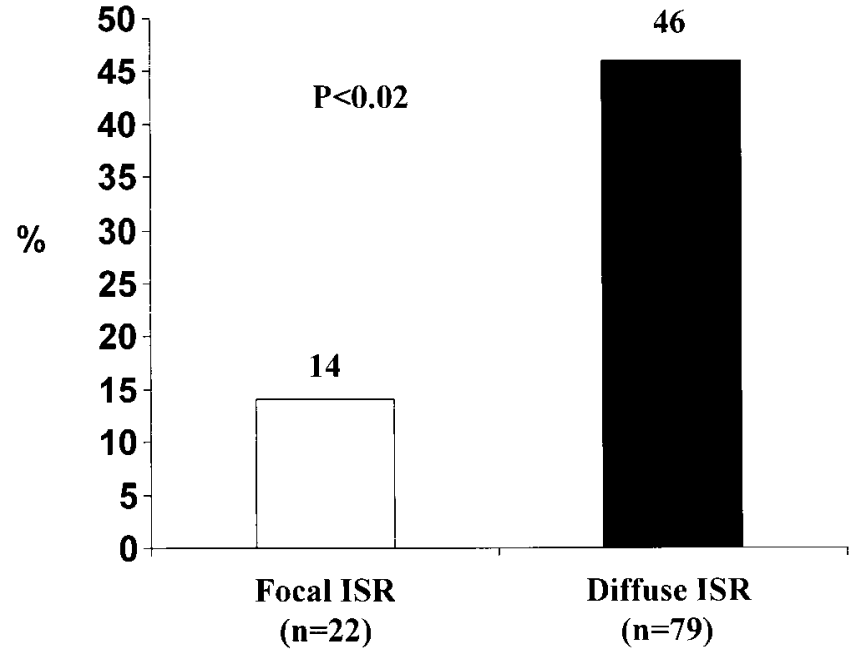

Fig. 3. Target lesion revascularization in two angiographic patterns of in-stent restenosis (ISR).

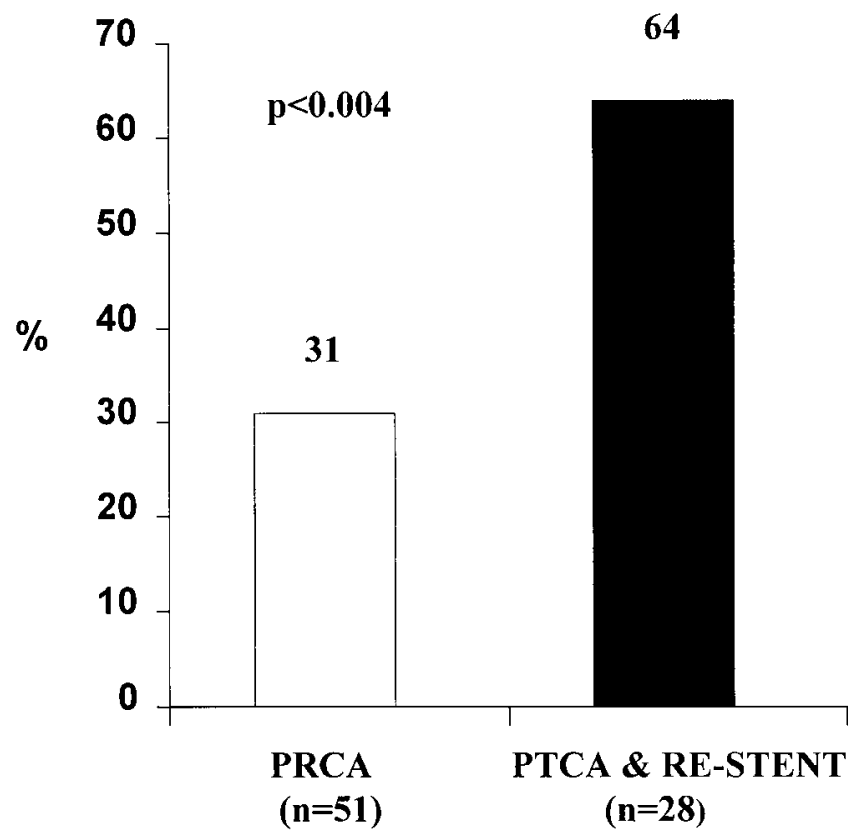

Fig. 4. Target lesion revascularization in diffuse in-stent restenosis after different devices.

\section{DISCUSSION}

Restenosis after successful stent placement, although less frequent than restenosis after PTCA, remains a major clinical challenge. Serial IVUS studies have shown that arterial remodeling is the predominant mechanism for restenosis after PTCA or atherectomy [7], but after stent implantation IVUS studies have shown that stents prevent vessel remodeling and in-stent restenosis occurs almost solely due to intimal hyperplasia $[7,15,19]$. Most of this intimal hyperplasia occurs between 3 and 6 months after intervention and after this period serial angiography and angioscopy have revealed intimal hyperplasia to thin out from 6 months to 3 years [20-22].

Our present study of 101 patients revealed diffuse ISR in the majority (78\%), after high-pressure balloon stent ( $\geq 3.0 \mathrm{~mm}$ Palmaz-Schatz) deployment. This was in contrast to an earlier report by Yokoi et al. $[9,23]$ revealing diffuse ISR occurring in $44 \%$ of cases while focal inside the stent occurred in $33 \%$ and focal at the stent borders in 23\%. This difference in the lower incidence of diffuse ISR in their study can be explained on the basis of clinical vs. angiographic follow-up between the two studies and also different types of stents used in their study. It is possible that diffuse in-stent restenosis tends to be more symptomatic due to greater lesion length of the exaggerated intimal hyperplasia compared to the focal type irrespective of the angiographic percent luminal stenosis [24]. In a recent report by Mehran et al. [10] of 166 Palmaz-Schatz ISR lesions, focal ISR occurred in $49 \%$, diffuse in $40 \%$, and total occlusions in $11 \%$. Many of these ISR lesions had undergone prior intervention and may explain the difference in angiographic patterns compared to our study, which included only the first-time ISR. Several studies have shown the various predictors of in-stent restenosis $[5,25,26]$. Patients with diabetes mellitus have a higher restenosis rate due to aggressive intimal hyperplasia, greater plaque burden, and remodeling that occurs after intervention [5]. These patients also tend to have diffuse ISR rather than focal ISR. Long lesions (type $\mathrm{C}$ of ACC/AHA classification), especially after multiple stent use, had diffuse ISR. The time interval for ISR is another important predictor for diffuse ISR. In our study, diffuse ISR occurred at an interval of $129 \pm 38$ days after stent deployment, compared to $161 \pm 47$ days for focal ISR. This intriguing observation, reported for the first time to our knowledge, may be a marker of aggressive intimal hyperplasia occurring soon after stent deployment. Early ISR occurring $\leq 90$ days of stent deployment has been shown to respond poorly to repeat intervention $[12,15]$. This early ISR represents an aggressive vascular response to injury caused by initial high-pressure stent implantation. Also, stents deployed for restenotic lesions had a higher incidence of diffuse ISR, indicating an exaggerated intimal hyperplastic response. Left anterior descending (LAD) artery lesions had a trend toward higher incidence of diffuse ISR when compared with non-LAD lesions. Similar findings observed by Carrozza et al. [6] showed LAD lesion location to have a higher restenosis rate. In our study, similar to earlier reports, small vessel diameter, lesion length, and use of multiple stents were associated with higher diffuse ISR [9,25]. Our observation of higher incidence of diffuse ISR after high inflation pressures $(>16 \mathrm{~atm})$ corroborates that higher balloon 
inflation pressure during initial stent deployment increases neointimal proliferation and TLR at follow-up $[27,28]$.

The angiographic pattern of ISR has an important bearing on the subsequent response to reintervention $[11,15,16]$. Treatment of ISR has very high procedural success rates $(\sim 99 \%)$ and low complication rates $(1 \%-$ $3 \%$ ), but long-term results are discouraging $[13,16]$. Diffuse ISR has been shown to have a higher TLR up to $85 \%$ after successful intervention [9-11,13,22]. Most of these studies involved PTCA as a treatment modality for ISR intervention $[9,13,29]$. Preliminary data have revealed ablative or debulking modalities like PRCA, excimer laser, or directional atherectomy to have better long-term outcome compared to PTCA in treatment of diffuse ISR [14-17]. This could be explained by the fact that ISR is solely due to intimal hyperplasia and PTCA causes plaque compression and extrusion [29] compared to plaque ablation by PRCA [14,15], excimer laser [16], or plaque removal by directional coronary atherectomy [17]. In our study, TLR after diffuse ISR was $46 \%$ compared to $14 \%$ in the focal type. This is in accordance with numerous published reports revealing a high subsequent TLR of $48 \%$ (range $21 \%-85 \%$ ) in diffuse ISR. PRCA had a lower TLR of $31 \%$ compared to $64 \%$ after PTCA and restenting in our series. Therefore, debulking should be preferred over balloon angioplasty or restent in the treatment of diffuse ISR. Based on these encouraging preliminary results, a randomized trial comparing PTCA with PRCA in treatment of diffuse ISR (ROSTER trial) has been started [30]. Recent reports using intracoronary radiation have also shown to decrease significantly neointimal proliferation and TLR in recurrent diffuse ISR [31], and soon may become the viable option either alone or in combination with debulking techniques [32].

\section{Study Limitations}

The present study has several important limitations, which should be considered before interpreting the final results. This study evaluated the first-time ISR of $\geq 3.0$ $\mathrm{mm}$ Palmaz-Schatz stent in one-vessel disease that presented clinically for intervention. It is possible that angiographic follow-up of all stent patients may yield a higher number of focal ISR than noted in the current study, because focal ISR may produce fewer symptoms. The study is only limited to Palmaz-Schatz stents and results may not be extrapolated to other stent designs, which may have different angiographic restenosis. Also, the type of interventions used was not standardized and left to the discretion of the interventionist. This represents a very early experience and hence it was appropriate at that time, not knowing the niche of individual device in specific ISR patterns. There was no angiographic follow-up after repeat intervention, which may underesti- mate the incidence of clinically silent recurrent angiographic ISR. Since the main aim of the present study was to evaluate the impact on clinical restenosis, all patients were closely followed for clinical events and recurrence. Finally, IVUS analysis was performed only in a handful of cases $(n=33)$ and therefore was not included in the analysis. High inflation pressures ( $>16 \mathrm{~atm})$, irrespective of the vessel size and lesion subset, may not accurately reflect its predictive value for subsequent diffuse in-stent restenosis.

The present study reports various factors that appear to be associated with aggressive intimal hyperplasia contributing to the occurrence of diffuse ISR. Therefore, modifications of identifiable factors at the time of initial stent deployment may lead to a lower incidence of diffuse ISR, such as spot stenting in long lesions, lower inflation pressures $(<16 \mathrm{~atm})$, and debulking prior to stenting. Interventional treatment for in-stent restenosis is safe but is associated with high TLR in the diffuse type. Rotational atherectomy appears to be more effective than other modalities in the treatment of diffuse in-stent restenosis and hence it should be preferred.

\section{REFERENCES}

1. Fischman D, Leon M, Baim D, et al. A randomized comparison of coronary-stent placement and balloon angioplasty in the treatment of coronary artery disease. N Engl J Med 1994;331:496-501.

2. Serruys P, de Jaegere P, Kiemeneij F, et al. A comparison of balloon expandable-stent implantation with balloon angioplasty in patients with coronary artery disease. N Engl J Med 1994;331:489-495.

3. Serruys S, Emanuelsson H, van der Giessen W, et al. Heparincoated Palmaz-Schatz stents in human coronary arteries: early outcome of the Benestent-II pilot study. Circulation 1996;93:412422.

4. Sawade Y, Nosaka H, Kimura T, Nobuyoshi M. Initial and six months outcome of Palmaz-Schatz stent implantation: stress/ benestent equivalent vs. nonequivalent lesions. J Am Coll Cardiol 1997;27(Suppl A):252A.

5. Kornowski R, Mintz G, Kent K, et al. Increased restenosis in diabetes mellitus after coronary intervention due to exaggerated intimal hyperplasia. Circulation 1997;95:1366-1369.

6. Carrozza J, Kuntz R, Levine M, et al. Angiographic and clinical outcome of intracoronary stenting: immediate and long term results from a large single center experience. J Am Coll Cardiol 1994;23:1051-1060.

7. Mintz G, Popma J, Pichard A, et al. Arterial remodeling after coronary angioplasty: a serial intravascular ultrasound study. Circulation 1996;94:35-43.

8. Komatsu R, Udea M, Naruko T, et al. Neointimal tissue response at sites of coronary stenting in humans: macroscopic, histological, and immunohistochemical analyses. Circulation 1998;98:224233.

9. Yokoi H, Kimura T, Nakagawa Y, Nosaka H, Nobuyoshi M. Long term clinical and quantitative angiographic follow-up after PalmazSchatz stent restenosis. J Am Coll Cardiol 1996;27(Suppl A): 224A.

10. Mehran R, Abizaid A, Mintz G, et al. Patterns of in-stent restenosis: classification and impact on subsequent target lesion revascularization. J Am Coll Cardiol 1998;31(Suppl A):141A. 
11. Sharma S, Rajawat Y, Kakarala V, Marmur J, Duvvuri S, Cocke T, Ambrose J. Angiographic pattern of in-stent restenosis after Palmaz-Schatz stent implantation. J Am Coll Cardiol 1997; 27(Suppl A):313A.

12. Reimers B, Moussa I, Akiyama T, et al. Long-term clinical follow-up after successful repeat percutaneous intervention for stent restenosis. J Am Coll Cardiol 1997;30:186-192.

13. Alfonsos F, Perez-Vizcayno MJ, Hernandez R, et al. Long term outcome and determinants of event-free survival in patients treated with balloon angioplasty for in-stent restenosis. Am J Cardiol 1999;83:1268-1270.

14. Dahl J, Radke P, Haager $P$, et al. Clinical and angiographic predictors of recurrent restenosis after percutaneous transluminal rotational atherectomy for treatment of diffuse in-stent restenosis. Am J Cardiol 1999;83:862-867.

15. Sharma S, Duvvuri S, Dangas G, Kini A, et al. Rotational atherectomy for in-stent restenosis: acute and long term results of first 100 cases. J Am Coll Cardiol 1998:32:1358-1367.

16. Mehran R. Mintz G, Satler L, et al. Treatment of in-stent restenosis with excimer laser coronary angioplasty: mechanisms and results compared with PTCA alone. Circulation 1997;96:2183-2189.

17. Dauerman H, Baim D, Cutlip D, et al. Mechanical debulking versus balloon angioplasty for the treatment of diffuse in-stent restenosis. Am J Cardiol 1999;82:277-284.

18. Reiber J, Serruys P. Quantitative coronary arteriography. In: Marcus ML, Schelbert HR, Skorton DJ, Wolf GL, editors. Cardiac imaging. Philadelphia: W.B. Saunders; 1991. p 211-280.

19. Gordon P, Gibson C, Cohen D, Carrozza J, Kuntz R, Baim D. Mechanism of restenosis and redilatation within coronary stents: quantitative angiographic assessment. J Am Coll Cardiol 1993;21: 1166-1174.

20. Kimura T, Yokoi H, Nakagawa Y, et al. Three-year follow-up after implantation of metallic coronary-artery stents. N Eng J Med 1996;334:561-566.

21. Asakura M, Ueda Y, Nanto S, et al. Remodeling of in-stent neointima, which became thinner and transparent over 3 years: serial angiographic and angioscopic follow-up. Circulation 1998; 97:2003-2006

22. Nibler N, Kastrati A, Elezi A, Walter H, Schulen H, Schomig A. Angiographic and clinical follow-up of patients with asymptomatic restenosis after coronary stent implantation. J Am Coll Cardiol 1998;31(Suppl A):65A.

23. Yokoi H, Tamura T, Kimura T, Nosaka H, Nobuyoshi M. Clinical and angiographic predictors of diffuse in-stent restenosis. J Am Coll Cardiol 1998;31(Suppl A):140A.

24. Uren N, Melin J, Bruyne B, Wijns W, Baudhuin T, Camici P. Relation between myocardial blood flow and the severity of coronary artery stenosis. N Engl J Med 1994;330:1782-1788.

25. Kastrati A, Schomig A, Elezi S, et al. Predictive factors of restenosis after coronary stent placement. J Am Coll Cardiol 1997;30:1428-1436.

26. Hoffman R, Mintz GS, Dussaillant GR, Popma JJ, Pichard Ad, Satler LS, Kent K, Griffin J, Leon M. Pattern of in-stent restenosis: a serial intravascular ultrasound study. Circulation 1996;94:12471254

27. Savage M, Fischman D, Douglas J, et al. The dark side of high pressure stent deployment. J Am Coll Cardiol 1997;29(Suppl A):368A.

28. Fernandez-Aviles F, Alonso J, Duran J, et al. High pressure increases late loss after coronary stenting. J Am Coll Cardiol 1997;29(Suppl A):369A.

29. Mehran R, Mintz G, Popma J. Mechanism and results of balloon angioplasty the treatment of in-stent restenosis. Am J Cardiol 1996;78:618-622.

30. Sharma S, Kini A, Duvvuri S, et al. Randomized trial of rotational atherectomy vs. balloon angioplasty for in-stent restenosis (ROSTER): interim analysis of 100 cases. Circulation 1998;98:I511.

31. Tierstein P, Massullo U, Jani S, et al. Catheter based radiotherapy to inhibit restenosis after coronary stenting. $\mathrm{N}$ Eng $\mathrm{J}$ Med 1997:336:1697-1703.

32. Waksman R. Endovascular brachytherapy: overcoming "practical obstacles." Am J Cardiol 1998;81:21E-26E. 